\title{
Detalhando o perfil de atividade dos jovens brasileiros que não estudam nem trabalham: o papel da busca por trabalho e dos afazeres domésticos ${ }^{*}$
}

\author{
Guilherme Cardoso** \\ Ana Hermeto***
}

Uma questão presente na literatura que trata dos jovens que não estudam nem trabalham é a carência da compreensão de suas características. 0 objetivo do presente estudo foi encontrar os determinantes do perfil dos jovens que se encontram nas diferentes categorias de condição ocupacional - trabalha e estuda; só trabalha; só estuda; nem estuda nem trabalha, mas procura emprego ("nem-nem" ativo); e nem estuda nem trabalha e nem procura emprego ("nem-nem" inativo) -, bem como buscar a relação entre o fato de os jovens encontrarem-se nas duas últimas categorias e a maior incidência de afazeres domésticos semanais em seus domicílios. Assim, com os dados da Pesquisa Nacional por Amostra de Domicílio de 2015, empregaram-se os métodos logit multinomial e de contagem (regressão de Poisson: incidence rate ratios e zero inflated). Os resultados mostram que o perfil de um jovem pertencente à condição ocupacional "nem-nem" ativo difere-se consideravelmente de outro classificado como "nem-nem" inativo, principalmente quanto ao gênero e à situação de residência. Relativamente às demais categorias, a carga horária de afazeres domésticos incide com maior frequência nos jovens "nem-nem" ativos e inativos, ao passo que estes também estão menos propensos a se pouparem das atividades domésticas. Mostrou-se de suma importância o discernimento entre ativos e inativos para a categoria dos que nem trabalham nem estudam e, a partir dos resultados encontrados, pode-se traçar indicativos de uma inatividade generalizada em parte dessa população, acendendo o alerta para a necessidade de políticas trabalhistas orientadas para o público jovem no Brasil.

Palavras-chave: Jovens. Condição ocupacional. Afazeres domésticos. Microeconometria.

\footnotetext{
* Os autores agradecem o parecer dos avaliadores anônimos da Rebep, imprescindível para os avanços trazidos na presente versão do trabalho.

** Centro de Desenvolvimento e Planejamento Regional (Cedeplar), Universidade Federal de Minas Gerais (UFMG), Belo Horizonte-MG, Brasil (scardoso.guilherme@gmail.com; ORCID: 0000-0002-0702-8992).

${ }^{\star \star \star}$ Centro de Desenvolvimento e Planejamento Regional (Cedeplar), Universidade Federal de Minas Gerais (UFMG), Belo Horizonte-MG, Brasil (ahermeto@cedeplar.ufmg.br; ORCID: 0000-0001-8331-1928).
} 


\section{Introdução}

Difundido na literatura nacional como "nem-nem", o termo empregado para caracterizar os jovens que não estudam nem trabalham pode ser assimilado internacionalmente à expressão not in education, employment or training (NEET). Inicialmente atrelada ao tempo de transição entre escola e trabalho, essa condição tem se tornado prolongada, complicada e individualizada (BYNNER et al., 2019; LÓPEZ BLASCO; MCNEISH, 2003). Ainda hoje há uma carência na compreensão do tamanho, características e distribuição geográfica, além do estereótipo acerca do comportamento desses jovens “nem-nem” (MAGUIRE, 2015).

Dois aspectos têm chamado a atenção da literatura nesse debate. Primeiro, a consideração de que a categoria "nem-nem" reflete apenas um grupo de desengajados na educação e no mercado de trabalho, quando, na prática, há evidências de que nem todos os jovens nessa condição estão em inatividade. Em segundo lugar, a dedicação aos afazeres domésticos como justificativa para os jovens encontrarem-se em condição "nem-nem”, algo especialmente verdadeiro para as mulheres.

Este artigo procura contribuir, para o caso brasileiro, justamente nesses dois aspectos, buscando os determinantes do perfil dos jovens em cada categoria de condição ocupacional. Além disso, procura-se averiguar se há relação entre encontrar-se na categoria "nem-nem" e a maior incidência de afazeres domésticos semanais por parte desses jovens. No primeiro caso, conta-se com o modelo logit multinomial e, para o segundo, empregam-se modelos de dados em contagem ("zero-inflated" Poisson), ambos usando os dados da Pesquisa Nacional por Amostra de Domicílio de 2015.

Existem muitas possibilidades de abordar esse tema empiricamente, que variam de acordo com o objetivo específico de investigação, muitas vezes intrínseco ao local de aplicação. Para o Brasil, Camarano et al. (2006), Almeida e Figueredo (2017) e Monteiro (2013), utilizando técnicas econométricas para explicar e mensurar as chances de os jovens encontrarem-se na condição "nem-nem”, verificam que o sexo, a cor, a condição na família e a localidade do domicílio são fatores determinantes. Os dois primeiros trabalhos analisam a população de 15 a 29 anos de idade e o terceiro, a de 19 a 24 anos. Para outros países latino-americanos, por meio de regressões logísticas, Corseuil, Santos e Foguel (2001) buscam comparar os fatores que determinam a escolha dos jovens entre as condições ocupacionais e destacam a influência positiva do background educacional nas condições ativas de trabalho e estudo.

No âmbito internacional, Bradley et al. (2020) investigam as variações espaciais e clusters nas taxas de desemprego juvenil e dos NEET e comparam os resultados de Itália, Espanha e Reino Unido. Os autores observam que, no geral, a oferta de trabalho dos jovens é sensível às condições do mercado, como a possibilidade de trabalho temporário ou de meio expediente. Samoilenko e Carter (2015) avaliam a duração de permanência de jovens na condição "nem-nem" na Nova Zelândia e comparam as características dos grupos de maior e menor tempo nessa situação. Por meio de modelos de propensity score matching 
e logit, o tempo na condição "nem-nem”, características familiares, coorte e indicador de qualidade da região de habitação foram as variáveis de interesse da investigação e, de acordo com seus resultados, o tempo de permanência na situação influencia positivamente na probabilidade de desemprego e inatividade futura.

Embora o tema não seja incipiente na literatura, em especial na internacional, este artigo contribui para o avanço da temática ao incorporar, para o caso brasileiro, a análise dos jovens de 15 a 29 anos que não estudam e não trabalham diferenciando-os entre grupos de inativos e ativos economicamente. Ademais, analisa-se a incidência dos afazeres domésticos para as categoriais abordadas.

A próxima seção descreve a metodologia adotada no estudo, seguida pela análise descritiva dos dados e a discussão dos resultados. Por fim, tecem-se as considerações finais.

\section{Metodologia}

São apresentados, aqui, os modelos empíricos empregados para os objetivos deste artigo. Por meio da Pesquisa Nacional por Amostra de Domicílio (PNAD) de 2015, do Instituto Brasileiro de Geografia e Estatística (IBGE), os aspectos estruturais estariam contemplados, visto que tal levantamento dispõe das variáveis necessárias para que os resultados obtidos sejam comparáveis com os outros trabalhos empíricos voltados para a mesma temática.

\section{Modelo logit multinominal ${ }^{1}$}

Os modelos logit multinomial estimam as probabilidades de ocorrência de um evento discreto condicionais a um conjunto de variáveis determinantes. Eles incorporam apenas efeitos das características individuais, ou seja, somente variáveis independentes que variam de acordo com os indivíduos, e não com as categorias assumidas pela variável dependente (CORSEUIL; SANTOS; FOGUEL, 2001). Em outras palavras, para o objetivo do trabalho, esse modelo parte do pressuposto de que as decisões de estudar ou não, trabalhar ou não e procurar ou não emprego são tomadas simultaneamente.

A variável dependente é uma variável discreta que assume cinco categorias $(j=1,2$, $3,4,5)$ que representam, respectivamente: trabalha e estuda; (só) trabalha; (só) estuda; não trabalha nem estuda, mas procura emprego; e não trabalha, não estuda e não procura emprego - os inativos. A "utilidade" que a $i$-ésima pessoa é derivada da $j$-ésima alternativa pode ser representada por $U_{i j}$. Supondo-se que essa utilidade seja uma função linear de $R$ fatores específicos aos indivíduos (variáveis independentes/determinantes), então a função utilidade deve ser escrita como:

$U_{i j}=\sum_{r=1}^{R} \beta_{j r} X_{i r}+\varepsilon_{i j}$

\footnotetext{
${ }^{1}$ Salvas as exceções devidamente referenciadas, esta seção foi inteiramente baseada em Melo (2012) e Cameron e Trivedi (2005).
} 
Na qual $\beta_{j r}$ é o coeficiente associado à $r$-ésima característica para $j$-ésima categoria assumida pela variável dependente.

Se os termos de erro $\varepsilon_{i j}$ forem idênticos e independentemente distribuídos (iid), com distribuição $F\left(\varepsilon_{i j}\right)=\exp \left[\exp \left(-\varepsilon_{i j}\right)\right]$, então a probabilidade de $j=m$ é dada por:

$\operatorname{Pr}\left(Y_{i}=m\right)=\frac{\exp \left(\beta_{m r} X_{i r}\right)}{\sum_{j=1}^{M} \exp \left(\beta_{j r} X_{i r}\right)}$

O logaritmo da razão de probabilidade de $j=m$ em relação a $j=k$ é:

$\log \left(\frac{\operatorname{Pr}\left(Y_{i}=m\right)}{\operatorname{Pr}\left(Y_{i}=k\right)}\right)=\sum_{r=1}^{R}\left(\beta_{m r}-\beta_{k r}\right) X_{i r}=Z_{\mathfrak{I}}-Z_{i k}$

Dessa forma, o logaritmo da razão de risco relativo (RRR) (isto é, o logaritmo da razão de probabilidade relativa da categoria $m$ em relação a $k$, ou $\log \left[\operatorname{prob}\left(Y_{i}=m\right) / p r o b\left(Y_{i}=k\right)\right]$ não depende das outras categorias que a variável $Y_{i}$ possa assumir. A razão de risco relativo pode ser calculada tomando o exponencial.

Novamente, considerando as categorias assumidas pela variável dependente deste exercício (1 - trabalha e estuda; 2 - (só) trabalha; 3 - (só) estuda; 4 - não trabalha nem estuda, mas procura emprego e 5 - não trabalha, não estuda e não procura emprego), se, da equação (3), $k=5$, o logaritmo da RRR da categoria 1 em relação à 5 , por exemplo, é: $\log \left(\frac{\operatorname{Pr}\left(Y_{i}=1\right)}{\operatorname{Pr}\left(Y_{i}=5\right)}\right)=\sum_{r=1}^{R} \beta_{1 r} X_{i r}=Z_{i 1}$

E o RRR é calculado como:

$$
\left(\frac{\operatorname{Pr}\left(Y_{i}=1\right)}{\operatorname{Pr}\left(Y_{i}=5\right)}\right)=\exp \left(\sum_{r=1}^{R} \beta_{1 r} X_{i r}\right)=\exp \left(Z_{i 1}\right)
$$

Para facilitar a interpretação da razão de riscos relativos, a mesma será convertida em incremento percentual, que fornece as chances de mudança da categoria base para a categoria analisada em função das variações das características dos indivíduos, da seguinte maneira: (RRR-1)*100. Em outras palavras, considerando o caso analisado neste exercício, o incremento percentual do coeficiente RRR deve ser interpretado como as chances de um indivíduo na categoria 3, por exemplo, relativamente a um indivíduo na categoria 5.

Nesta aplicação de modelo, temos como variável dependente, portanto, a condição ocupacional, cuja categoria de referência é “nem-nem" inativo; e as variáveis independentes, apresentadas no Quadro 1, têm o objetivo de captar características multidimensionais dos indivíduos, conforme fizeram alguns dos trabalhos empíricos apresentados na introdução deste artigo. São elas: sexo; raça; idade; anos de estudo; estado matrimonial (cônjuge); condição de filho no domicílio de residência (filho); macrorregião (Nordeste, Sudeste, Sul e Centro-Oeste), com referência à região Norte; meio urbano; e região metropolitana. 
QUADRO 1

Variáveis explicativas empregadas no modelo logit multinomial

\begin{tabular}{|cc|}
\hline Variáveis & Descrição \\
\hline Sexo & Feminino/masculino (referência) \\
Raça & Não branco/branco (referência) \\
Idade & 15 a 29 anos \\
Anos de estudo & 0 a 15 anos \\
\hline Cônjuge & Condição na família com relação à pessoa \\
Filho & de referência no domicílio (referência) \\
\hline Macrorregião & Referência: região Norte \\
Urbano & Urbano/rural \\
RM & Região metropolitana \\
\hline
\end{tabular}

Fonte: Elaboração dos autores.

Modelos de contagem - regressão de Poisson: razões de incidência relativa (incidence rate ratios - IRR) e de "inflação de zeros"

Para encontrar evidência empírica para a relação positiva entre ser um jovem em condição “nem-nem” e a elevada carga horária semanal de afazeres domésticos, utilizaram-se modelos de dados em contagem cuja especificação foi conforme a apresentada em Cameron e Trivedi (2005). Esse modelo é o mais adequado considerando que a distribuição do número de horas dedicadas a afazeres domésticos semanalmente pelos jovens aparenta seguir a distribuição de Poisson, com concentração de respostas nulas (zero) e perda de frequência à medida que se aumenta o número de respostas.

O modelo estocástico natural para dados de contagens se baseia na distribuição de Poisson para a ocorrência do evento de interesse.

$\operatorname{Pr}[Y=y]=\frac{e^{-\mu} \mu^{y}}{y !}$

Em que $\mu$ é a intensidade de ocorrência e y corresponde a uma variável aleatória discreta.

Para um modelo de Poisson, assumimos que nossa variável de resposta é uma variável de contagem, sendo que cada elemento tem o mesmo tempo de observação e a variância da variável de resposta é relativamente próxima da média da variável de resposta. A propriedade de equidispersão da distribuição de Poisson garante que os dois primeiros momentos (média e variância) sejam iguais a $\mu$. A forma usual consiste na utilização da parametrização de média exponencial:

$\mu_{i}=\exp \left(x^{\prime}{ }_{i} \beta\right), i=1, \ldots, N$

Para qualquer modelo com média condicional exponencial, a diferenciação assegura que:

$\frac{\partial E[y \vee x]}{\partial x_{j}}=\beta_{j} \exp \left(x^{\prime} \beta\right)$

Em que o escalar $x_{j}$ denota o $j$-ésimo regressor. Por exemplo, se $\hat{\beta}=0,25$ e $\exp \left(x^{\prime}{ }_{i} \hat{\beta}_{j}\right)=3$, então uma mudança unitária no $j$-ésimo regressor aumenta a y esperada em 0,75 unidade. Se $x_{j}$ é medido em escala logarítmica, $\beta_{j}$ é uma elasticidade. 
A regressão de Poisson com “inflação de zeros" (zero-inflated Poisson - ZIP) é usada para modelar dados de contagem que tenham um excesso de contagens zero. Além disso, a teoria sugere que os zeros em excesso são gerados por um processo separado a partir dos valores de contagem e que os zeros em excesso podem ser modelados independentemente, como no caso em que se trata de jovens que não exercem atividades dométicas e daqueles que exercem. Assim, o modelo ZIP possui duas partes: um modelo de contagem de Poisson e o modelo logit binário para estimar o excesso de zeros.

Nesta aplicação de modelo, temos como variável dependente, portanto, a carga de afazeres domésticos dividida entre os valores acima de zero (modelo IRR), seguida da ausência total de afazeres domésticos entre os jovens de 15 a 29 anos (modelo ZIP). As variáveis independentes, apresentadas no Quadro 2, são as mesmas do modelo logit multinomial, com a inclusão de renda familiar per capita e tamanho da família, além da condição do jovem entre trabalha, estuda, “nem-nem” ativo e "nem-nem” inativo (trabalha e estuda é a categoria de referência). Retirou-se o controle das macrorregiões.

QUADRO 2

Variáveis explicativas empregadas nos modelos de Poisson

\begin{tabular}{|cc|}
\hline Variáveis & Descrição \\
\hline Sexo & Feminino/masculino (referência) \\
Raça & Não branco/branco (referência) \\
Idade & 15 a 29 anos \\
Anos de estudo & 0 a 15 anos \\
Condição ocupacional & Condição ocupacional do jovem (referência: trabalha e estuda) \\
\hline Cônjuge & Condição na família com relação à pessoa \\
Filho & de referência no domicílio \\
Tamanho da família & Número de membros no domicílio \\
Renda & Renda familiar per capita (R $\$$ ) \\
\hline Urbano & Local de residência (urbano/rural) \\
\hline
\end{tabular}

Fonte: Elaboração dos autores.

Para concluir a seção metodológica e introduzir a próxima, dedicada à análise descritiva dos dados, faz-se necessário expor as principais estatísticas descritivas das variáveis explicativas dos modelos empregados. Controlando para jovens de 15 a 29 anos de idade, a Tabela 1 apresenta a média e o desvio-padrão das variáveis empregadas no modelo logit multinomial e de Poisson. 
TABELA 1

Média e desvio-padrão das variáveis explicativas empregadas no modelo logit multinomial e de Poisson, para jovens de 15 a 29 anos

Brasil - 2015

\begin{tabular}{ccc}
\hline Variáveis & Média & Desvio-padrão \\
\hline Sexo (\%) & 51,0 & $(0,4999)$ \\
Raça (\%) & 39,0 & $(0,4876)$ \\
Anos de estudo & 6,83 & $(4,8715)$ \\
\hline Chefe da família (\%) & 19,0 & - \\
Cônjuge (\%) & 16,0 & - \\
Filho (\%) & 55,0 & $(1,4848)$ \\
Outros (\%) & 10,0 & $(4.370,07)$ \\
Tamanho da família & 3,52 & - \\
Renda (em R\$) & $3.154,05$ & - \\
Norte (\%) & 17,0 & - \\
Nordeste (\%) & 30,0 & - \\
Sudeste (\%) & 28,0 & $(0,3526)$ \\
Sul (\%) & 14,0 & $(0,4830)$ \\
\hline Centro-Oeste (\%) & 11,0 & \\
Urbano (\%) & 85,0 & \\
RM (\%) & 37,0 & \\
\hline
\end{tabular}

Fonte: IBGE. PNAD (2015). Elaboração dos autores.

Nota: Para variáveis binárias, como sexo e raça, os números mostram a representatividade da categoria de referência; no caso, homens e brancos.

A Tabela 1 sintetiza informações relevantes com relação ao objeto de estudo do presente trabalho, isto é, os jovens de 15 a 29 anos de idade, como é o caso da distribuição regional e urbana. A próxima seção dedica-se a explorar as heterogeneidades dessa faixa etária, com relação às condições ocupacionais e, principalmente, quanto à escolaridade, renda e ao viés de gênero.

\section{Análise descritiva dos dados}

Utilizando a base de dados da Pesquisa Nacional por Amostra de Domicílio (PNAD) de 2015, é importante pontuar, de início, a considerável participação demográfica da população de investigação do trabalho: os jovens de 15 a 29 anos representavam, em 2015 , aproximadamente $24 \%$ da população brasileira. Com relação à condição ocupacional desses jovens, os dados indicam que aproximadamente $11 \%$ estavam na categoria dos que trabalham e estudam, $39 \%$ na de só trabalham, $26 \%$ na de só estudam, $9 \%$ não estudam nem trabalham, mas procuram emprego ("nem-nem" ativo) e $15 \%$ na condição de "nem-nem" inativo.

O Gráfico 1 mostra a composição dos jovens entre as diferentes condições ocupacionais, com destaque para a distinção entre ativos e inativos no mercado de trabalho para aqueles que não trabalham e nem estudam. Para o presente estudo, optou-se pela distinção entre "nem-nem" ativos e "nem-nem" inativos, sendo o primeiro grupo composto pelos jovens 
que não trabalham e nem estudam, mas que procuram emprego, e o segundo por aqueles que não exercem nenhuma das atividades e não procuram emprego.

\section{GRÁFICO 1}

Distribuição dos jovens de 15 a 29 anos, segundo situação de trabalho e estudo Brasil - 2015

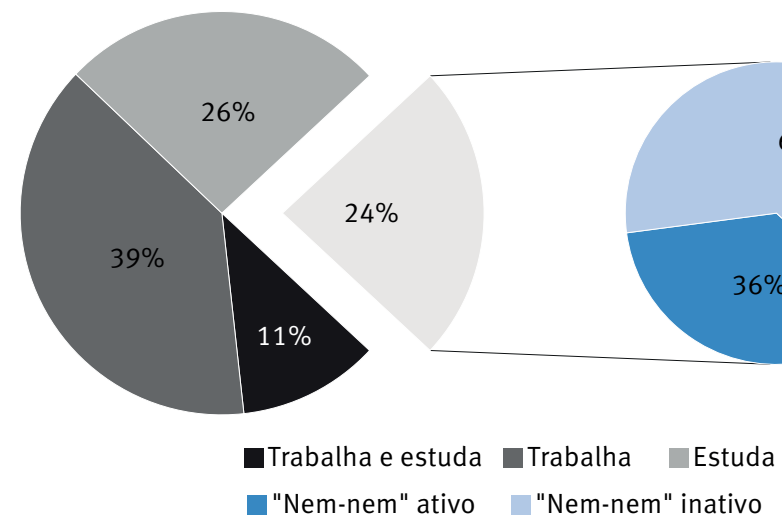

Fonte: IBGE. PNAD (2015). Elaboração dos autores.

Como se pode notar no Gráfico 1, 24\% dos jovens de 15 a 29 anos não trabalham nem estudam, todavia, mais de um terço desses jovens são considerados ativos, ou seja, estão procurando emprego e contribuindo para que a condição seja passageira.

Devido ao fato de haver muitas categorias a serem analisadas, optou-se, na presente seção, assim como nos resultados, focar apenas nos grupos "nem-nem" ativo e "nem-nem" inativo, para melhor fomentar a discussão e evitar fuga da centralidade de compreender o fenômeno dos "nem-nem" nas suas distinções entre ativos e inativos, a grande contribuição da pesquisa.

O Gráfico 2 mostra a distribuição dos jovens entre as categorias de condições ocupacionais, segundo a idade.

Na parte superior do gráfico, observa-se que, ao longo da distribuição etária dos jovens, as categorias referentes aos "nem-nem" ativos e inativos ganham volume expressivo a partir dos 17 anos e permanece equilibrada a partir dos 18 anos - ambas partem de aproximadamente $5 \%$ da população, aos 15 anos, e alcançam em torno de 30\% dos 18 aos 29 anos. Essa composição é dividida entre aproximadamente $20 \%$ de inativos e $10 \%$ de jovens que não estudam e nem trabalham, mas procuram emprego.

O Gráfico 3 mostra a distribuição da escolaridade dos jovens entre as condições ocupacionais.

A perda de participação dos jovens na condição "nem-nem" ativo e "nem-nem" inativo é progressiva com relação aos anos de estudo. No entanto, observa-se, em ambas as categorias, uma concentração de jovens com 11 e 15 anos de estudo, períodos que correspondem, respectivamente, aos ensinos médio e superior completos, indicando a ociosidade dos jovens após a conclusão de suas formações. 


\section{GRÁFICO 2}

Distribuição dos jovens de 15 a 29 anos, por idade, segundo condições ocupacionais Brasil - 2015

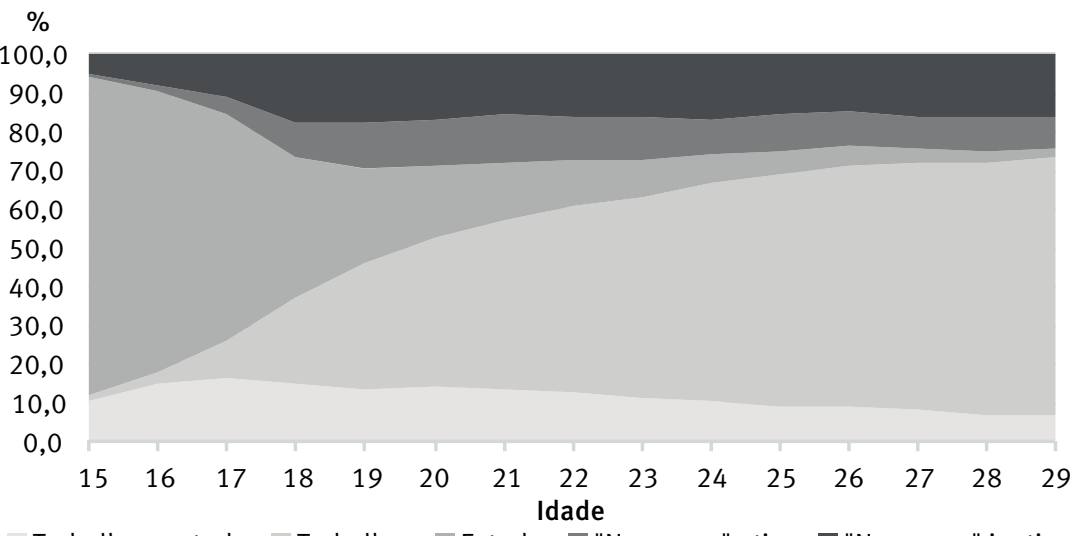

Fonte: IBGE. PNAD (2015). Elaboração dos autores.

\section{GRÁFICO 3}

Distribuição dos jovens de 15 a 29 anos, por anos de estudo, segundo condições ocupacionais Brasil - 2015

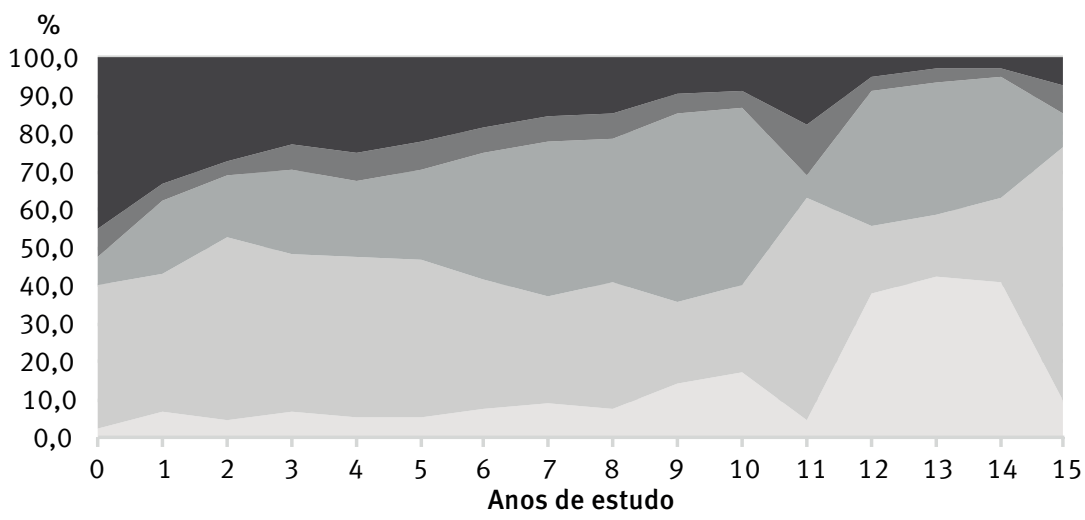

Trabalha e estuda Trabalha Estuda "Nem-nem" ativo " "Nem-nem" inativo

Fonte: IBGE. PNAD (2015). Elaboração dos autores.

No âmbito regional, vale mencionar que a proporção da distribuição de cada condição ocupacional se assemelha entre os meios rural e urbano. A PNAD (2015) mostra que, para todas as categorias, os jovens habitantes do meio rural representam cerca de $10 \%$ a $20 \%$ do total. Quanto ao papel desempenhado pelos jovens dentro de suas respectivas famílias, foi possível analisar, pelos dados da PNAD (2015), a distinção por gênero da condição familiar dos jovens "nem-nem" ativos e inativos. Os homens nessa situação são, em sua maioria, filhos; já entre as mulheres predominam as cônjuges. 
Compreender a condição financeira familiar dos jovens é também de suma importância. A Tabela 2 exibe a renda familiar total e per capita média dos jovens que se encontram em cada categoria ocupacional.

TABELA 2

Renda familiar total e per capita média dos jovens de 15 a 29 anos, segundo condição ocupacional Brasil - 2015

\begin{tabular}{lcc} 
& & Em reais \\
\hline \multicolumn{1}{c}{ Condição ocupacional } & Renda familiar total & Renda per capita \\
\hline Trabalha e estuda & 3.935 & 1.179 \\
Só trabalha & 3.138 & 1.020 \\
Só estuda & 3.311 & 881 \\
"Nem-nem" ativo & 1.901 & 538 \\
"Nem-nem" inativo & 1.962 & 546 \\
\hline
\end{tabular}

Fonte: IBGE. PNAD (2015). Elaboração dos autores.

Nota-se que os jovens "nem-nem" ativos e inativos estão inseridos em famílias cujo rendimento médio domiciliar é mais baixo em relação às famílias que tinham jovens em outras categorias. Além do fato dos que trabalham contribuírem diretamente para a elevação da renda familiar, é preciso considerar a possibilidade de endogeneidade advinda da condição financeira familiar.

Para a questão dos jovens em condição “nem-nem” ativos e inativos e a ocupação com afazeres domésticos, a justificativa do uso do método de dados em contagem, por meio da regressão de Poisson e de "inflação de zeros", deve-se ao fato de a distribuição das horas ocupadas com afazeres domésticos entre os jovens apresentar distribuição de Poisson, com concentração de observações no início da contagem de ocorrências do evento em análise - no caso, horas despendidas semanalmente em afazeres domésticos. Os dados da Tabela 3 indicam que essa atividade, entre os jovens de 15 a 29 anos, apresenta viés de gênero, resultado encontrado também em Brasil (2013).

TABELA 3

Distribuição dos jovens de 15 a 29 anos, por sexo, segundo número de horas dedicadas semanalmente a afazeres domésticos

Brasil - 2015

\begin{tabular}{cccc}
\hline Horas & Mulheres (\%) & Homens (\%) & N \\
\hline 0 & 22,0 & 78,0 & 27.190 \\
a 7 a 14 & 39,0 & 61,0 & 18.596 \\
15 a 21 & 59,0 & 41,0 & 15.046 \\
22 a 30 & 78,0 & 22,0 & 11.823 \\
31 a 40 & 89,0 & 11,0 & 5.966 \\
Acima de 40 & 94,0 & 6,0 & 3.111 \\
\hline
\end{tabular}

Fonte: IBGE. PNAD (2015). Elaboração dos autores.

Além da notável diferença na concentração de zeros, indicando que os homens estão mais propensos a não exercerem atividade doméstica alguma, o número de mulheres 
sobressai ao dos homens ao longo de toda a escala que indica o intervalo do número de horas dedicadas ao exercício semanal de afazeres domésticos.

A Tabela 4 exibe as médias e desvios-padrão do número de horas dedicadas aos afazeres domésticos por sexo e condição ocupacional dos jovens.

TABELA 4

Média e desvio-padrão do número de horas dedicadas semanalmente a afazeres domésticos entre os jovens de 15 a 29 anos, por sexo, segundo condição ocupacional

Brasil - 2015

\begin{tabular}{lcccccc}
\hline \multicolumn{1}{c}{$\begin{array}{c}\text { Condição } \\
\text { ocupacional }\end{array}$} & \multicolumn{5}{c}{ Média (desvio-padrão) } \\
\cline { 2 - 7 } & \multicolumn{2}{c}{ Geral } & \multicolumn{2}{c}{ Homens } & \multicolumn{1}{c}{ Mulheres } \\
\hline Trabalha e estuda & 6,9 & $(8,63)$ & 3,9 & $(5,87)$ & 10,5 & $(9,96)$ \\
Só trabalha & 8,8 & $(11,02)$ & 4,5 & $(6,65)$ & 15,5 & $(1,29)$ \\
Só estuda & 8,9 & $(10,28)$ & 4,8 & $(6,91)$ & 12,5 & $(1,13)$ \\
"Nem-nem" ativo & 14,9 & $(14,88)$ & 6,3 & $(8,35)$ & 22,3 & $(15,27)$ \\
"Nem-nem" inativo & 19,3 & $(17,95)$ & 4,5 & $(7,83)$ & 24,8 & $(17,53)$ \\
\hline
\end{tabular}

Fonte: IBGE. PNAD (2015). Elaboração dos autores.

Na mesma direção das informações da Tabela 3, constata-se que jovens entre 15 e 29 anos que se dedicam ao trabalho e ao estudo são os que, em média, menos contribuem com os afazeres domésticos em suas residências. A categoria dos "nem-nem" inativos é a que, em média, mais contribui; no entanto, é a que apresenta o maior desvio-padrão, sendo assim o grupo menos homogêneo em termos de horas dedicadas a afazeres domésticos. Novamente, destaca-se o exacerbado viés de gênero entre as categorias de condição ocupacional, em que a diferença entre a carga de afazeres dos homens e a das mulheres aumenta de acordo com o grau de inatividade.

Justifica-se a investigação da relação dos afazeres domésticos com o fenômeno "nem-nem” pelo fato de que o primeiro é um trabalho não pago muitas vezes negligenciado. Faz-se necessário pontuar que a questão de gênero é o elemento central para compreender a relação dos fenômenos.

Esta seção contribuiu para que se conhecessem os dados a serem trabalhados nas inferências econométricas apresentadas nos modelos que serão descritos a seguir; após o conhecimento das características dos dados, é possível compreender melhor os modelos e esperar determinados comportamentos de algumas variáveis, dado o objetivo e contexto das regressões estimadas, apresentadas anteriormente.

\section{Resultados}

Modelo logit multinomial - comparação relativa das características das condições ocupacionais entre os jovens de 15 a 29 anos

Para comparar os determinantes relativos do perfil dos jovens entre as cinco condições ocupacionais - estudo, trabalho, ambas as atividades, nenhuma das atividades ("nem-nem" ativo e inativo) -, utilizou-se o modelo logit multinomial. Com finalidades analíticas, 
considerou-se como referência a categoria 5, correspondente aos jovens "nem-nem" inativos. Assim, os resultados serão interpretados relativamente a essa categoria. A Tabela 5 exibe as razões de risco relativa (RRR) de cada variável entre as categorias em análise.

TABELA 5

Resultados da regressão logística multinomial - razão de risco relativa (RRR). Variável dependente: condição ocupacional; categoria de referência: "nem-nem” inativo Brasil - 2015

\begin{tabular}{ccccc}
\hline Variáveis & Trabalha e estuda & Só trabalha & Só estuda & "Nem-nem" ativo \\
\hline Sexo & 3,39 & 5,2 & 1,87 & 2,37 \\
Raça & $(38,36)$ & $(63,21)$ & $(21,67)$ & $(-25,31)$ \\
Idade & 1,08 & $0,96^{*}$ & 1,23 & 0,83 \\
& $(2,47)$ & $(-1,69)$ & $(6,97)$ & $(-5,17)$ \\
Anos de estudo & 0,86 & 1,11 & 0,6 & 1,02 \\
Cônjuge & $(-32,7)$ & $(32,29)$ & $(-85,28)$ & $(-5,09)$ \\
& 1,41 & 1,15 & 1,29 & 1,13 \\
Filho & $(56,78)$ & $(39,87)$ & $(48,38)$ & $(-23,21)$ \\
& 0,43 & 0,56 & 0,5 & 0,6 \\
Nordeste & $(-14,68)$ & $(-17,08)$ & $(-10,75)$ & $(-10,34)$ \\
& 1,32 & 0,62 & $(1,6,26)$ & 1,11 \\
Sudeste & $(6,26)$ & $(-14,81)$ & 0,88 & $(-2,41)$ \\
& 0,98 & 1,13 & $(-3,42)$ & 1,28 \\
Sul & $(-0,56)$ & $(3,8)$ & 0,82 & $(-5,26)$ \\
& 1,12 & 1,66 & $(-4,87)$ & 1,58 \\
Centro-Oeste & $(-2,43)$ & $(14,22)$ & 0,76 & $(-9,41)$ \\
& 1,63 & 2,06 & $(-5,49)$ & 1,35 \\
Urbano & $(-8,95)$ & $(16,52)$ & 0,94 & $(-4,84)$ \\
& 1,4 & 1,54 & $(-1,2)$ & 1,08 \\
RM & $(6,05)$ & $(9,63)$ & 1,6 & $(-1,21)$ \\
& 0,9 & 0,92 & $(12,16)$ & 2,2 \\
& $(-2,44)$ & $(-2,75)$ & 1,22 & $(-14,33)$ \\
& 1,03 ns & 1,01 ns & $(6,84)$ & $(-6,25$ \\
& $(1,05)$ & $(0,23)$ & & $(-69)$ \\
\hline
\end{tabular}

Fonte: Elaboração dos autores com base nos resultados da regressão. Entre parênteses, valor z.

* - Significativa a 90\% de confiança; ns - Não significativa. Todas os demais resultados são significativos a $95 \%$ de confiança.

Podemos observar que a probabilidade de homens estarem na categoria dos que trabalham e estudam, vis-à-vis a dos “nem-nem” inativos, é 3,39 vezes maior do que a probabilidade de as mulheres trabalharem e estudarem vis-à-vis serem inativas. A probabilidade relativa de os homens encontrarem-se na categoria dos que só trabalham é ainda maior (5,2 vezes mais provável do que as mulheres). A comparação de gênero somente diminui para a categoria dos que só estudam; no entanto, observa-se que a chance de homens estarem em tal categoria é 1,87 vez maior, em relação à dos “nem-nem” inativos, do que a de mulheres. Homens possuem 2,37 vezes mais chances de não estudarem nem trabaIharem, mas serem economicamente ativos, vis-à-vis serem inativos, do que as mulheres.

A probabilidade relativa de um indivíduo branco trabalhar e estudar vis-à-vis se enquadrar nos inativos é 1,08 vez maior do que a de um indivíduo negro. Essa vantagem desaparece analisando a categoria dos que só trabalham, relativamente à dos inativos, e aumenta para aqueles que só estudam (brancos com 1,23 vez mais chance). Jovens brancos 
também estão menos propensos a serem “nem-nem” ativos, comparados à categoria dos inativos, do que jovens negros.

Interpretamos a RRR da variável idade como o impacto de um ano a mais de vida na categoria em análise relativamente à de referência. A RRR menor do que 1 nas categorias 1 e 3 (trabalham e estudam; e só estudam) é bastante plausível: conforme mencionado anteriormente, quanto mais idade tem o jovem, maior é a chance de não trabalhar e nem estudar, relativamente a trabalhar e estudar ou só estudar. A interpretação é semelhante para a variável referente à escolaridade do jovem, que, conforme mostrado nas estatísticas descritivas dos dados, quanto maior a escolaridade, menor é a participação dos jovens na categoria "nem-nem" inativo.

$\mathrm{Na}$ análise referente à variável condição na família, cuja comparação é feita com a pessoa de referência no domicílio, observa-se que a probabilidade de jovens com o papel de cônjuges dentro de suas famílias estarem na categoria dos que trabalham e estudam, vis-à-vis a dos "nem-nem" inativos, é aproximadamente 1,57 vez menor do que a probabilidade da pessoa de referência. Com exceção da comparação "nem-nem" ativos e inativos, que indica que jovens na condição de cônjuges estão 1,4 vez mais propensos a serem inativos do que se enquadrarem no status estabelecido como "nem-nem" ativos, relativamente à pessoa de referência no domicílio, a probabilidade dos jovens em condição de cônjuge é inferior à das pessoas de referência na comparação com todas as categorias de condição ocupacional, relativamente aos "nem-nem" inativos. Filhos estão, respectivamente, 1,3 e 2,6 vezes mais propensos a trabalhar e estudar e somente estudar, vis-à-vis "nem-nem" inativos, do que uma pessoa de referência no domicílio. Tal condição familiar leva desvantagem à pessoa de referência apenas para a condição de somente trabalhar, relativamente a nenhuma das atividades ( $38 \%$ de chance a menos).

Para a análise da influência regional nas condições ocupacionais dos jovens brasileiros, se estabelece a região Norte do país como macrorregião de referência. Assim, pode-se inferir que jovens habitantes do Sudeste, Sul e Centro-Oeste estão mais propensos a trabalhar e estudar, somente trabalhar e serem categorizados como "nem-nem" vis-à-vis ser inativo, do que os jovens da região Norte. Juntam-se a essas três regiões os jovens do Nordeste na comparação relativa com as categorias "só trabalha", "só estuda" e "nem-nem" ativo. Quando se tem a comparação trabalhar vis-à-vis ser "nem-nem” inativo, conclui-se que jovens do Norte estão em desvantagem perante as outras regiões; jovens da região vizinha, Centro-Oeste, por exemplo, estão 1,5 vez mais propensos a trabalharem, vis-à-vis serem classificado como "nem-nem" inativo, do que jovens nortistas. Jovens habitantes de zona urbana estão em vantagem perante os moradores de área rural quando se considera a possibilidade de somente estudar ou não estudar e nem trabalhar, mas ser economicamente ativo vis-à-vis ser inativo. Estes estão em desvantagem diante dos jovens do meio rural na comparação relativa entre somente trabalhar e exercer ambas as atividades vis-à-vis ser "nem-nem" inativo. 
Para os habitantes da região metropolitana de seus estados, observa-se resultado semelhante ao obtido para a variável urbano. Os habitantes das metrópoles possuem 1,22 e 1,25 vez mais chance de somente estudar e ser "nem-nem" ativo, respectivamente, vis-à-vis serem classificados como “nem-nem” inativos, do que seus vizinhos interioranos.

Assim, após a estimação dos resultados do modelo logit multinomial, para cada categoria de condição ocupacional em análise, pode-se traçar perfis de jovens mais ou menos propensos a estarem sob a condição de "nem-nem" inativos. Com base nos resultados, conclui-se que homens brancos são pouco propensos à condição "nem-nem" inativo.

Observa-se, portanto, que os determinantes do perfil de um jovem pertencente à condição ocupacional "nem-nem" ativo diferem-se consideravelmente daqueles referentes ao jovem classificado como "nem-nem” inativo, principalmente quanto ao gênero e à situação de residência: mulheres e moradores do meio rural, por exemplo, estão muito mais propensos a acatarem o fato de viver sem estudos e sem trabalho e, assim, se classificarem como "nem-nem" inativos.

Modelo de dados de contagem - regressão de Poisson e de "inflação de zeros" (ZIP): a incidência da carga de afazeres domésticos sobre os jovens.

Conforme apresentado inicialmente e contextualizado na seção anterior, o que os modelos de regressão de Poisson e de “inflação de zeros" buscam medir aqui é a relação entre a condição ocupacional dos jovens e o número de horas dedicadas a afazeres domésticos. Ao controlar para variáveis individuais e domiciliares, pretende-se obter o valor mais acurado da incidência em que cada condição ocupacional é exposta a variável resposta, relativamente à categoria dos que trabalham e estudam. No modelo ZIP, as interpretações possuem como referência o risco de não se dedicar a afazeres domésticos, isto é, ser um dos "zeros" na resposta de tal variável. A Tabela 6 traz os resultados do modelo. A regressão de Poisson é feita para aqueles que exercem alguma quantidade positiva de afazeres domésticos semanalmente e o modelo ZIP, para aqueles que não exercem.

Conforme indicam os resultados da regressão de Poisson, destaca-se a discrepância para a variável gênero, em que a execução de afazeres domésticos possui incidência $43 \%$ maior sobre as mulheres do que sobre os homens. Jovens negros também estão mais propensos à realização das tarefas, em relação aos brancos.

No que se refere às condições ocupacionais, os resultados são intuitivos e indicam que jovens em todas as categorias estão mais propensos a despenderem mais tempo na execução de afazeres domésticos do que aqueles que trabalham e estudam. É interessante notar que os jovens na categoria "nem-nem" ativo, que não estudam e nem trabalham, mas procuram emprego, estão mais propensos à incidência de afazeres domésticos do que aqueles em condição similar, porém não procuram emprego, relativamente à categoria de referência (trabalha e estuda). 
TABELA 6

Resultados da regressão de Poisson - razões de incidência relativa (IRR). Características influentes na carga positiva de afazeres domésticos e modelo ZIP: fatores que influenciam a ausência de afazeres domésticos entre os jovens de 15 a 29 anos

Brasil - 2015

\begin{tabular}{|c|c|c|c|}
\hline Horas dedicadas a afazeres domésticos & IRR & Erro-padrão & Z \\
\hline Constante & 1,34 & 0,13 & 273,50 \\
\hline Sexo & 0,57 & 0,00 & $-201,75$ \\
\hline Raça & 0,96 & 0,00 & $-16,77$ \\
\hline Idade & 1,02 & 0,00 & 47,39 \\
\hline Anos de estudo & 0,99 & 0,00 & $-12,54$ \\
\hline Cônjuge & 1,14 & 0,00 & 43,79 \\
\hline Filho & 0,72 & 0,00 & $-93,04$ \\
\hline Tamanho da família & 1,02 & 0,00 & 19,99 \\
\hline Renda familiar per capita & 0,9999 & 0,00 & $-47,89$ \\
\hline Urbano & 0,95 & 0,00 & $-18,13$ \\
\hline Trabalha & 1,07 & 0,00 & 14,34 \\
\hline Estuda & 1,25 & 0,01 & 47,79 \\
\hline "Nem-nem" ativo & 1,45 & 0,01 & 71,66 \\
\hline "Nem-nem" inativo & 1,54 & 0,01 & 92,38 \\
\hline Ausência de afazeres domésticos & Odds ratio (1) & Erro-padrão & Z \\
\hline Constante & 0,04 & 0,08 & $-40,97$ \\
\hline Sexo & 5,4 & 0,02 & 90,43 \\
\hline Raça & 1,17 & 0,02 & 9,24 \\
\hline Idade & 1,22 & 0 & 7,38 \\
\hline Anos de estudo & 0,97 & 0 & $-10,23$ \\
\hline Cônjuge & 0,82 & 0,04 & $-5,55$ \\
\hline Filho & 2,68 & 0,03 & 36,24 \\
\hline Tamanho da família & 1,1 & 0,01 & 16,76 \\
\hline Renda familiar per capita & 1,00006 & 0 & 9,37 \\
\hline Urbano & 1,11 & 0,03 & 4,24 \\
\hline Trabalha & 1,14 & 0,03 & 4,57 \\
\hline Estuda & 0,78 & 0,03 & $-8,18$ \\
\hline "Nem-nem" ativo & 0,56 & 0,04 & $-14,18$ \\
\hline "Nem-nem" inativo & 0,92 & 0,04 & $-2,27$ \\
\hline
\end{tabular}

Fonte: Elaboração dos autores com base nos resultados da regressão.

(1) A interpretação em odds ratio se dá pela exponenciação dos coeficientes obtidos na regressão. Assimilada ao termo "razão de chances", é a medida que descreve as chances de sucesso associadas a um grupo em relação a outro.

Nota: Todos os resultados são significativos a $95 \%$ de confiança.

Jovens que se dedicam apenas ao trabalho estão $14 \%$ mais propensos, com relação aos que trabalham e estudam, a não se dedicarem a afazeres domésticos durante a semana. Jovens que só estudam estão $22 \%$ menos propensos, relativamente, a não realizarem tarefas domésticas. Embora os membros das categorias "nem-nem" ativos e inativos estejam menos propensos a se pouparem de afazeres domésticos, relativamente aos que trabalham e estudam, a propensão dos membros da primeira categoria é menor, sendo, portanto, a chance de um indivíduo "nem-nem" inativo contribuir com afazeres domésticos menor do que a dos ativos. 
De modo geral, os resultados adicionam conteúdo àquilo destacado em Brasil (2013), com relação à associação do sexo feminino com o número de horas de afazeres domésticos, e em Camarano et al. (2006) e Menezes et al. (2013), principalmente quanto ao risco de permanência na condição e inatividade generalizada daqueles que não estudam, não trabalham e nem procuram emprego.

\section{Considerações finais}

0 debate sobre a categoria "nem-nem" ganhou visibilidade no Brasil no período recente, em especial devido a um expressivo contingente populacional nessa categoria. 0 estudo dos jovens brasileiros nessa condição é, portanto, não apenas relevante e atual, mas também imperativo para se pensarem políticas públicas que ampliem a qualificação profissional e a inserção dos jovens no mercado de trabalho. 0 contexto de crise econômica e reformas tocantes ao mercado de trabalho que o país experimenta reforça a importância desse debate, em especial porque os jovens compõem uma categoria muito afetada no mercado de trabalho em períodos recessivos.

0 presente estudo contribui para o entendimento da realidade dos jovens brasileiros de 15 a 29 anos que não trabalham e não estudam, explorando as diferenças entre aqueles economicamente ativos, que procuram emprego no mercado de trabalho, e os inativos. Tal discernimento mostrou-se essencial para que se avançasse no tema a nível nacional, constatado que a maior preocupação para com os jovens em condição de desocupação está na possibilidade de ausência de perspectivas, o que levaria ao estado permanente da condição.

Para além das análises descritivas da base de dados - que de forma expositiva muito contribuíram para o conhecimento das características de cada categoria de condição ocupacional entre os jovens -, o modelo logit multinomial traçou o perfil de jovens mais ou menos propensos a estarem sob a condição de "nem-nem" inativo, relativamente às demais categorias ocupacionais. Na comparação direta entre as categorias "nem-nem" ativos e "nem-nem" inativos, observou-se que os determinantes do perfil de um jovem pertencente ao primeiro grupo diferem-se consideravelmente do jovem do segundo grupo, principalmente quanto ao gênero e à situação de residência, apontando a necessidade de explorações adicionais sobre a questão regional e de gênero no estudo dos jovens em tais condições ocupacionais.

Preocupou-se com o fato de muitos desses jovens, apesar de não se dedicarem ao trabalho nem aos estudos e serem economicamente inativos, estarem ocupados com uma carga horária relativamente maior de afazeres domésticos. Para as mulheres, a incidência semanal dessas atividades é muito superior à dos homens, assim como a representatividade das que não exercem nenhuma atividade doméstica é muito inferior. Enfatiza-se a necessidade de explorar ainda mais o papel do gênero na análise entre os jovens que não trabalham e nem estudam, como Hirata (2018), Kergoat (2003), Bartholo et al. (2019), Cyrino (2009) e Contatore et al. (2019), que, de maneira geral, investigam a divisão sexual do trabalho 
e o fato de a naturalização do cuidado como atributo feminino ter peso determinante na inatividade econômica das mulheres.

De modo geral, conforme exposto na análise descritiva dos dados, há um certo alívio de quantidade de afazeres para aqueles jovens sem trabalho e afastados da educação formal mas que procuram emprego; no entanto, os resultados obtidos no modelo ZIP nos levam a crer que o fato de ser economicamente ativo - indivíduo disposto a deixar a condição de "nem-nem" - não o alivia de ocupar-se com algum afazer doméstico, de modo que um jovem nesta categoria está mais propenso a realizar alguma atividade do que os inativos. Conforme ressaltado nos resultados do modelo ZIP, pode ser um indicativo de uma "inatividade generalizada" daqueles jovens que não estudam nem trabalham e nem procuram emprego.

Atualmente, o Brasil sofre com os impactos generalizados da pandemia provocada pela Covid-19, sinalizando que o fenômeno dos "nem-nem" pode se intensificar, tendo em vista a elevada taxa de desemprego. Isso torna ainda mais relevante a busca pela compreensão das características desses jovens. Apesar de não abordar especificamente o papel de políticas públicas voltadas para essa população, o presente trabalho serve de subsídio para tais ações, uma vez que explora de maneira multivariada os determinantes e desdobramentos da condição "nem-nem" no país. Os resultados encontrados sugerem necessidade de maior aprofundamento da investigação quanto às questões de gênero e de influência da situação de residência urbano e rural. Existe também a limitação de não se debruçar sobre o tipo e a qualidade do trabalho exercido pelos jovens recém-ingressantes no mercado de trabalho, tendo em vista o atual contexto de reforma trabalhista no país; tampouco sobre as condições e incentivos ao ingresso no sistema superior de ensino. Esses podem, no entanto, servir de motivação para agendas de pesquisa futuras, passíveis ainda de potenciais desdobramentos por meio da atualização da análise.

\section{Referências}

ALMEIDA, J. B. S. A. de; FIGUEIREDO, A. M. R. População nem-nem: uma análise a partir dos dados da PNAD 2012. Revista de Estudos Sociais, v. 19, n. 38, p. 106-129, jul. 2017. Disponível em: http:// periodicoscientificos.ufmt.br/ojs/index.php/res/article/view/4942. Acesso em: 15 out. 2019.

BARTHOLO, L.; PASSOS, L.; FONTOURA, N. Bolsa Família, autonomia feminina e equidade de gênero: o que indicam as pesquisas nacionais? Cadernos Pagu, Campinas, n. 55, 2019. Disponível em: http://www.scielo.br/scielo.php?script=sci_arttext\&pid=S0104-83332019000100514\&lng =en\&nrm=iso. Acesso em: 15 dez. 2020.

BRADLEY, S.; MIGALI, G.; NAVARRO PANIAGUA, M. Spatial variations and clustering in the rates of youth unemployment and NEET: a comparative analysis of Italy, Spain and the UK. Journal of Regional Science, v. 60, n. 5, p. 1074-1107, 2020. Disponivel em: https://doi.org/10.1111/ jors.12501. Acesso em: 11 set. 2020.

BRASIL. Ministério do Desenvolvimento Social e Combate à Fome. Os jovens que não estudam nem trabalham no Brasil: discussão conceitual, caracterização e evolução de 2001 a 2011. Brasília, 2013. (Estudo Técnico, n. 03/2013). 
BYNNER, J.; CHISHOLM, L.; FURLONG, A. Youth, citizenship and social change in a European context. Routledge, 2019.

CAMARANO, A. A.; KANSO, S.; MELLO, J. L.; ANDRADE, A. Estão fazendo a transição os jovens que não estudam, não trabalham e não procuram trabalho? In: CAMARANO, A. A. (org.). Transição para a vida adulta ou vida adulta em transição? Rio de Janeiro: Ipea, 2006.

CAMERON, A. C.; TRIVEDI, P. K. Econometrics: methods and applications. Cambridge, UK: Cambridge University Press, 2005. Disponivel em: http://www.centroportici.unina.it/centro/ Cameron\&Trivedi.pdf. Acesso em: 11 out. 2019.

CONTATORE, O. A.; MAlfitAnO, A. P. S.; BARROS, N. F. de. Por uma sociologia do cuidado: reflexões para além do campo da saúde. Trabalho, Educação e Saúde, Rio de Janeiro, v.17, n. 1, 2019. Disponível em: http://www.scielo.br/scielo.php?script=sci_arttext\&pid=S198177462019000100510\&lng=en\&nrm=iso. Acesso em: $15 \mathrm{dez} .2020$.

CORSEUIL, C. H.; SANTOS, D. D.; FOGUEL, M. N. Decisões críticas em idades críticas: a escolha dos jovens entre estudo e trabalho no Brasil e em outros países da América Latina. Rio de Janeiro: Ipea, 2001. (Texto para Discussão, n. 0797). Disponível em: http://www.ipea.gov.br/portal/index. php?option=com_content\&view=article\&id=4029. Acesso em 11 out. 2019.

CYRINO, R. Trabalho, temporalidade e representações sociais de gênero: uma análise da articulação entre trabalho doméstico e assalariado. Sociologias, Porto Alegre, ano 11, n. 21, p. 66-92, jan./jun. 2009.

HIRATA, H. Gênero, patriarcado, trabalho e classe. Revista Trabalho Necessário, v. 16, n. 29, p. 14-27, 2018. Disponível em: https://doi.org/10.22409/tn.16i29.p4552.

KERGOAT, D. Divisão sexual do trabalho e relações sociais de sexo. Trabalho e cidadania ativa para as mulheres: desafios para as políticas públicas. São Paulo: Coordenadoria Especial da Mulher, 2003. p. 55-63.

LÓPEZ BLASCO, A.; MCNEISH, W. (ed.). Young people and contradictions of inclusion: towards integrated transition policies in Europe. Policy Press, 2003.

MAGUIRE, S. NEET, unemployed, inactive or unknown - why does it matter? Educational Research, v. 57, n. 2, p. 121-132, 2015. Disponível em: https://www.tandfonline.com/doi/full/10.1080/001 31881.2015.1030850. Acesso em: 11 out. 2019.

MELO, L. M. C. de. Mobilidade sócio-ocupacional e mobilidade espacial: diferenciações entre hierarquias urbanas para o mercado de trabalho formal, Brasil, 2000-2009. Tese (Doutorado em Economia) - Centro de Desenvolvimento e Planejamento Regional, Universidade Federal de Minas Gerais, Belo Horizonte, 2012. Disponível em: https://repositorio.ufmg.br/handle/1843/ AMSA-8WZH4P. Acesso em: 11 out. 2019.

MENEZES FILHO, N. A.; LEE, M. K. H.; KOMATSU, B. K. Mudanças na situação de estudo e trabalho dos jovens no Brasil. Insper - Instituto de Ensino e Pesquisa, 2013. (Policy Paper, n. 8). Disponivel em: https://www.insper.edu.br/wp-content/uploads/2018/09/Mudancas_situacao_ jovens_2013.pdf. Acesso em: 11 out. 2019.

MONTEIRO, J. Quem são os jovens nem-nem? Uma análise sobre os jovens que não estudam e não participam do mercado de trabalho. FGV/Ibre, 2013. (Texto para Discussão, n. 34). FGV/Ibre, 2013. Disponivel em: http://bibliotecadigital.fgv.br/dspace;/handle/10438/11661. Acesso em: 11 out. 2019.

SAMOILENKO, A.; CARTER, K. Economic outcomes of youth not in education, employment or training (NEET). New Zealand Treasury, 2015. (Working Paper, n. 15/01). Disponivel em: https:// econpapers.repec.org/paper/nztnztwps/15_2f01.htm. Acesso em: 15 nov. 2019. 


\title{
Sobre os autores
}

Guilherme Cardoso é mestre e doutorando em Economia pelo Centro de Desenvolvimento e Planejamento Regional (Cedeplar), da Universidade Federal de Minas Gerais (UFMG).

Ana Hermeto é professora associada da Universidade Federal de Minas Gerais (UFMG). Coordenadora do Grupo de Pesquisas em Economia e Demografia da Estratificação Social (Pedes) e diretora de Fomento à Pesquisa da PRPq/UFMG.

\section{Endereço para correspondência}

\section{Guilherme Cardoso}

Universidade Federal de Minas Gerais, Faculdade de Ciências Econômicas, Centro de Desenvolvimento e Planejamento Regional

Av. Antônio Carlos, 6627, Pampulha

31270-901 - Belo Horizonte-MG, Brasil

\section{Ana Hermeto}

Universidade Federal de Minas Gerais, Faculdade de Ciências Econômicas, Centro de Desenvolvimento e Planejamento Regional

Av. Antônio Carlos, 6627, Pampulha

31270-901 - Belo Horizonte-MG, Brasil

\begin{abstract}
Detailing the activity profile of Brazilian young people who do not study or work: the role of job seeking and household chores

One issue in the literature regarding youngsters who neither study nor work (NEET) is the lack of understanding regarding their characteristics and the misdiagnosis of their stereotype. The aim of this study was to find the determinants of certain characteristics of young people in different occupational condition categories -work and study; just work; just study; NEET and seeking work (active NEET) and NEET not seeking work (inactive NEET), and to seek a correlation between the last two categories and the higher incidence of weekly household chores in the home. Thus, with data from the 2015 National Household Sample Survey (Pnad), we used the Logit Multinomial and Counting methods (Poisson regression: Incidence Rate Ratios and Zero Inflated). The results showed that the profile of a young person belonging to the active NEET occupational condition differs considerably from that of those classified as inactive NEETs, mainly regarding gender and housing area. With regard to the other categories, the workload of domestic chores is more frequent in active and inactive NEETs, who are also less likely to be exempt from domestic chores. The distinction between active and inactive NEET was extremely important for the "neither work nor study" category and, based on the results, it is possible to identify traces of generalized inactivity in this population, raising the alarm regarding the need for labor policies aimed at young people in Brazil.
\end{abstract}

Keywords: Young people. Occupational condition. Household chores. Microeconometrics. 


\section{Resumen}

Detalles del perfil de actividad de los jóvenes brasileños que no estudian ni trabajan: el papel de la búsqueda de empleo y de las tareas del hogar

Un tema en la literatura que trata con jóvenes que no estudian ni trabajan (ninî) es la falta de comprensión de sus características y el diagnóstico erróneo de su estereotipo. El objetivo del presente estudio fue encontrar los determinantes de los perfiles de los jóvenes que se encuentran en las diferentes categorías de condición ocupacional: trabaja y estudia, solo trabaja, solo estudia, ni estudia ni trabaja pero busca trabajo (niní activo) y ni estudia ni trabaja ni busca trabajo (niní inactivo), y buscar la relación del hecho de que los jóvenes están en las dos últimas categorías con la mayor incidencia de tareas domésticas semanales en sus hogares. Por lo tanto, con los datos de la Encuesta Nacional de Muestras de Hogares (PNAD) de 2015 utilizamos los métodos de logit multinomial y Poisson. Los resultados mostraron que el perfil de una persona joven que pertenece a la condición ocupacional activa niní difiere considerablemente de los clasificados como niní inactivos, principalmente en relación con el género y el área de vivienda. En relación con las otras categorías, la carga de trabajo de las tareas domésticas es más frecuente en los jóvenes niní activos e inactivos, mientras que también tienen menos probabilidades de salvarse de las actividades domésticas. Era sumamente importante discernir entre activos e inactivos para la categoría de quienes ni trabajan ni estudian y, con base en los resultados encontrados, rastrear indicios de inactividad generalizada en parte de esta población para encender la alerta de la necesidad de políticas laborales dirigidas a los jóvenes en Brasil.

Palabras clave: Jóvenes. Condición ocupacional. Tareas del hogar. Microeconometría.

Recebido para publicação em 24/12/2019 Aceito para publicação em 21/06/2021 